\title{
Réponses d'un aquifère fissuré de haute montagne à la fusion nivale Morzine-Avoriaz, Haute-Savoie
}

\author{
D. Lhomme \\ Laboratoire de Géologie Appliquée, Univ. Paris VI
}

M. Dzikowski \& G. Nicoud

Laboratoire de Géologie Structurale et Appliquée, Univ. de Savoie

\section{E. Naffrechoux}

Laboratoire de Chimie et d'Ingénierie de l'environnement, Univ. de Savoie

\begin{abstract}
Les études sur la fusion nivale en zones alpines sont nombreuses. Elles concernent généralement la prévision des crues sur de grands bassins versants dans le but d'améliorer la gestion des cours d'eau. Le suivi du stock neigeux fait appel à des méthodes variées telles que, la mesure des paramètres nivoclimatiques, le bilan énergétique et la télédétection appliquée sur de grands bassins [1]. L'étude réalisée ici sur le bassin de Chavanette (Morzine-Avoriaz) concerne les réponses à la fusion nivale, d'un aquifère calcaire et fissuré, au niveau d'une source pérenne d'altitude $(1850 \mathrm{~m})$ alimentée par un petit bassin versant dont la totalité de la surface reste enneigée sur la période étudiée. Le système est donc composé par le couvert neigeux, les zones d'infiltration et un aquifère. Il implique une approche hydrogéologique et nivoclimatique.
\end{abstract}

\section{PRÉSENTATION DU SITE}

C'est sur la commune de Morzine, dans le Chablais hautsavoyard, qu'est équipé le site de Chavanette (fig. l). Situé à 20 kilomètres au Sud du Léman, ce versant d'exposition Nord-Est s'étend entre la frontière suisse et les habitations de la station de sport d'hiver d'Avoriaz. Ces points culminants sont à des altitudes supérieures à 2300 mètres entre la Pointe de Vorlaz à l'Est $(2346 \mathrm{~m})$ et les Hauts Forts au Sud-Ouest (2 $372 \mathrm{~m})$. A la côte $1740 \mathrm{~m}$ se trouve le lac d'Avoriaz qui a pour bassin versant topographique le bassin de Chavanette dont la superficie totale est de $3 \mathrm{~km}^{2}$. Le réseau hydrographique temporaire est essentiellement actif en période de fonte au printemps ou lors de fortes pluies quand il n'est pas couvert de neige. Aucun aménagement ne permet de connaître les fluctuations de son débit. Tous les drains de surface convergent en amont du lac d'Avoriaz qui se situe dans une zone endoréique.

En période d'étiage hivernal, la source de Chavanette située à 1850 mètres d'altitude est une des principales ressources du réseau d'alimentation en eau potable. Son bassin versant topographique (surface : $0,92 \mathrm{~km}^{2}$ ) fait donc

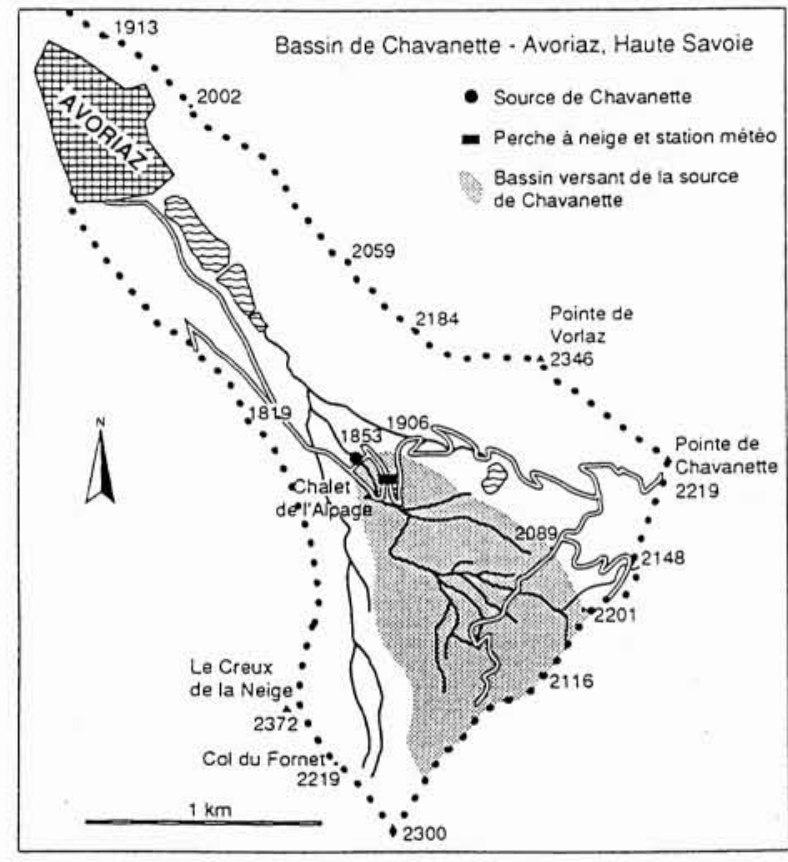

1. Le site expérimental de Chavanette.

plus particulièrement l'objet de cette étude. Contrairement à la plupart des ruisseaux du bassin, la source de Chavanette ne tarie jamais.

Du point de vue géologique, le site de Chavanette appartient entièrement à la « Nappe de la Brèche » du Chablais, formation hectométrique calcareo dolimitique, affectée par une fracturation cassante. La dépression du bassin de Chavanette semble être creusée dans la sousunité lithologique des Schistes et Calcaires inférieurs (Lias). Il s'agit de schistes calcaires dans lesquels sont intercalés des niveaux de calcaires bréchiques. 
Tableau 1. - Equipement scientifique du site. Période de mesure et pas d'enregistrement

\begin{tabular}{|c|c|c|c|c|c|}
\hline & & PAS & $1991-92$ & $1992-93$ & $1993-94$ \\
\hline \multirow{2}{*}{$\begin{array}{l}\text { Température } \\
\text { alt. : } 1850 \mathrm{~m}\end{array}$} & de l'air & $2 \mathrm{~h}$ & oui & oui & oui \\
\hline & du sol à $-10 \mathrm{~cm} /-80 \mathrm{~cm}$ & hebdo & oui/non & oui/non & oui/oui \\
\hline \multirow{3}{*}{$\begin{array}{l}\text { Nivologie } \\
\text { (sondage) } \\
\text { alt. : } 1850 \mathrm{~m}\end{array}$} & TEL (tout ou rien) & hebdo & oui & oui & non \\
\hline & Température de la neige & hebdo & oui & oui & non \\
\hline & Hauteur de neige & hebdo & oui & oui & oui \\
\hline \multirow{3}{*}{$\begin{array}{l}\text { Hydrologie } \\
\text { (source) } \\
\text { alt. : } 1850 \mathrm{~m}\end{array}$} & Température de l'eau & $2 \mathrm{~h}$ & oui & oui & oui \\
\hline & Conductivité de l'eau & $2 \mathrm{~h}$ & oui & oui & oui \\
\hline & Débit & $2 \mathrm{~h}$ & non & non & oui \\
\hline
\end{tabular}

\section{II — EQUIPEMENT ET MESURES SUR LE SITE}

L'équipement scientifique est concentré autour de la source captée de Chavanette (altitude $1850 \mathrm{~m}$ ). Pour des raisons techniques et climatiques, les mesures effectuées durant l'hiver et le printemps sur la période 1990-1994 ne sont pas continues. Le tableau I résume l'ensemble des paramètres mesurés.

\section{III $\square$ CARACTÉRISTIQUES NIVO- CLIMATIQUES}

Les deux hivers 1991-1992 et 1992-1993 ont été marqués par de courtes périodes de fusion qui se sont manifestées par la présence d'eau liquide dès lors que la température moyenne du manteau atteint les valeurs de $-1{ }^{\circ} \mathrm{C}$ à $-2{ }^{\circ} \mathrm{C}$ (fig. 2). A partir de mars, le mécanisme d'altération du stock nival est un processus irréversible. Le manteau garde en mémoire son altération à travers l'évolution des cristaux de neige et la présence de glace qui augmente sa densité. Entre les premières hausses de température de l'air et la transformation du stock nival en écoulement, il faut un laps de temps nécessaire pour que la capacité de rétention en eau des strates de neige soit atteinte.

Les valeurs négatives de la température du sol, mesurées à $-10 \mathrm{~cm}$ et à $-80 \mathrm{~cm}$ de la surface témoignent d'un blocage de l'infiltration de l'eau en début de période de fonte. Cependant, de simples sondages effectués simultanément dans les dépressions topographiques du site, ont révélé la présence, sous le couvert neigeux, de poches d'eau stagnante à des températures légèrement positives. Ceci implique que l'infiltration soit localisée et non uniforme sur tout le bassin.

\section{IV $\square$ EVOLUTION DES PARAMÈTRES À LA SOURCE DE CHAVANETTE}

L'étiage hivernal est caractérisé par des valeurs constantes des paramètres (fig. 3). Le débit d'étiage est de l'ordre de $3 \mathrm{l} / \mathrm{s}$, conductivité et température conservent des valeurs

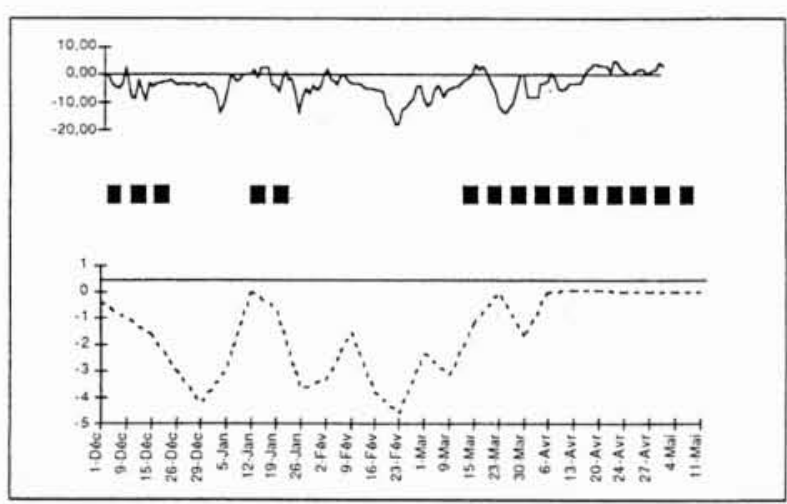

2. Evolution de la température extérieure en ${ }^{\circ} \mathrm{C}$ (trait plein), présence d'eau liquide au sein du manteau (barres noires) et température moyenne du manteau en ${ }^{\circ} \mathrm{C}$ (Tirets) en 1993.

respectives de $330 \mu \mathrm{S} / \mathrm{cm}$ et $3,5^{\circ} \mathrm{C}$. Les premières crues de fin de tarissement entraînent une diminution de la température de $0,5^{\circ} \mathrm{C}$ sans baisse de conductivité. Les crues ultérieures caractérisent la fusion généralisée du couvert neigeux. Elles sont généralement précédées par un pic de conductivité. Les variations de ces deux paramètres comme les variations de débit sont assez bien corrélées avec des températures extérieures positives.

\section{DÉCOMPOSITION DE L'HYDRO- GRAMME DE CRUE}

La décomposition d'hydrogramme de crue permet de mettre en évidence l'importance relative des différentes composantes de l'écoulement. Les études sur la décomposition des hydrogrammes de crues de fonte sont généralement effectuées sur le réseau hydrographique ([2], [3] et [4]). Les méthodes employées sont fondées sur les propriétés physico-chimiques ([5] et [6]) de l'eau ou utilisent des modèles hydrodynamiques de récession de crue. Dans le cadre de notre étude, les deux composantes de l'écoulement à dissocier à la source sont l'eau de fonte qui a atteint 


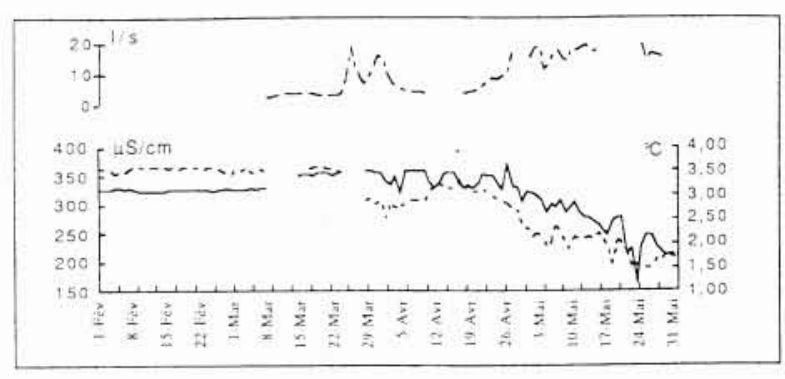

3. Exemple d'évolution des paramètres physicochimiques à la source de Chavanette en 1994. Tiretspoints : débit ; Tirets: température; Trait plein : conductivité.

l'aquifère après infiltration et l'eau de récession du système (volume dynamique de la réserve).

\subsection{Décomposition thermique}

Nous avons procédé à partir des équations de conversion des flux de chaleur (1) et de matière (2) qui permettent d'obtenir l'équation (3) :

$$
\begin{aligned}
Q \cdot T & =Q^{r} \cdot T^{r}+Q^{n} \cdot T^{n} \\
Q & =Q^{r}+Q^{n} \\
Q^{n} & =Q \frac{T-T^{r}}{T^{n}-T^{r}}
\end{aligned}
$$

où $Q$ est le débit total, $T$ la température de l'eau de source, $Q^{n}$ le débit d'eau de fusion, $Q^{r}$ le débit de vidange de la réserve, $T^{n}$ la température de la neige et enfin $T^{r}$ la température de la réserve. La température de la réserve $T^{r}$ est celle observée à l'étiage soit $3,5^{\circ} \mathrm{C}, T^{n}$ est la température de fusion de la neige soit $0^{\circ} \mathrm{C}$.

\subsection{Modèle de récession}

Le modèle de récession que nous avons considéré comme étant le plus performant a été utilisé par de nombreux auteurs ([7], [8] et [9]). Le modèle permet de simuler les ruptures de pente qui surviennent lors de la récession et pouvant être liées à des variations de la géométrie de l'aquifère [10].

Le débit de récession $Q_{d+1}^{r}$ est déduit du débit total ici égal à $Q_{d}^{r}$ de la veille, il s'exprime de la façon suivante :

$$
Q_{d+1}^{r}=\rho\left(Q_{d}^{r}\right)^{e} \quad \text { avec } e \leqslant 1
$$

La composante d'eau de fusion $Q^{n}$ est alors obtenue en soustrayant au débit total, le débit de récession soit :

$$
Q_{d}^{n}=Q_{d}-Q_{d}^{r}
$$

L'optimisation des paramètres par moindres carrés sur la période de décrue du mois d'avril 1994, exempte de terme pluvieux et leur validation sur la période du 8 au 31 mars 1994 ont abouti au modèle de récession suivant:

$$
Q_{d+1}^{r}=1.44\left(Q_{d}^{r}\right)^{0.68}
$$

avec un coefficient de corrélation est de 0,99 .

\subsection{Comparaison des différentes décompositions}

Pour les apports liés à la fusion généralisée (à partir d'avril), les résultats obtenus par les deux approches sont similaires (fig. 4) mais diffèrent pour les premières crues de fin de tarissement (fin mars). Le décalage de l'ordre du jour entre les débits déterminés, par le modèle de récession (transfert de pression) et la méthode thermique, implique un transfert retardé d'eau de fusion. Il apparaît également lors de la décrue que la décomposition thermique génère artificiellement une composante d'eau de fonte à $0{ }^{\circ} \mathrm{C}$ pour conserver le flux de chaleur. Au cours du temps, la température de l'eau de la réserve diminue lors des apports de fusion. Le retour progressif de la température de l'eau de la réserve à sa valeur d'étiage doit être due à un transfert plus lent lorsque le volume hydrodynamique diminue. Dans le cas de la fusion généralisée, ce retard tend à s'atténuer.

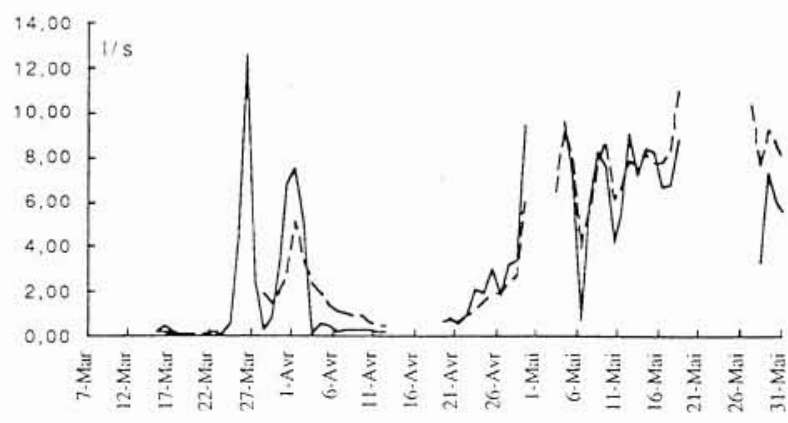

4. Comparaison des débits de fusion en 1994, par la méthode thermique (Tirets) et à partir du modèle de récession (Trait plein).

\section{DISCUSSION}

La nature géologique du terrain, l'étude des hydrogrammes ainsi que les variations rapides des paramètres physicochimiques de la source (température et conductivité) pendant la période de fusion caractérise un milieu fissuré ouvert avec des circulations rapides. Ces résultats associés à l'interprétation des décompositions du débit total impliquent :

- une faible réserve qui ne permet pas de créer un effet tampon lors d'apport d'eau de fusion ;

- des transferts rapides empêchant tout rééquilibrage thermique avec l'encaissant lors des crues. 


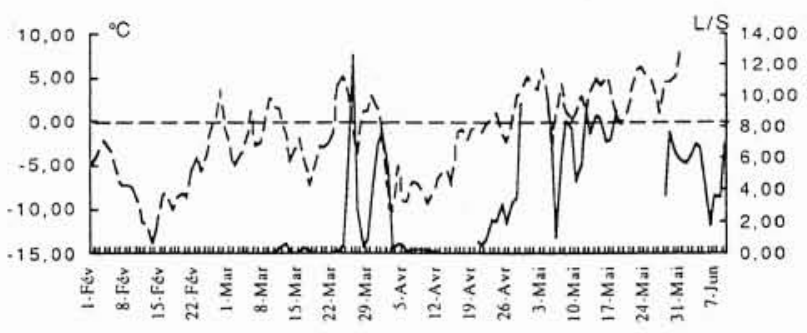

5. Evolution des températures extérieures journalières moyennes (Tirets) et du débit lié à la fusion (Trait plein).

\section{RELATION TEMPÉRATURE EXTÉ- RIEURE-DÉBIT DE LA SOURCE}

Les difficultés opératoires liées à un environnement de haute montagne imposent l'emploi d'un nombre limité de paramètres dans la conception d'un modèle de simulation des débits. Les modèles degré-jour répondent à cet impératif puisqu'ils ne nécessitent, pour les plus simples d'entre eux, que la connaissance de la température de l'air.

Les modèles de type degré jour permettent de simuler la composante d'eau de fusion de l'hydrogramme de crue ; de manière générale ils se présentent sous la forme :

$$
Q^{n}=F\left(T-T_{0}\right) S
$$

$Q^{n}$ est le débit de fusion.

$F$ est le facteur degré-jour en $1 . \mathrm{s}^{-1} \cdot{ }^{\circ} \mathrm{C}^{-1}$ ou la quantité d'écoulement produit pour un accroissement de $1{ }^{\circ} \mathrm{C}$. Le facteur degré-jour peut être un terme constant ou tenir compte de facteurs qui agissent sur la fonte ([8], [11] et [12]).

$T$ est généralement la température moyenne de l'air. $\left(T-T_{0}\right)$ est donc le nombre de degrés au-dessus de $T_{0}$, température au-dessus de laquelle la neige fond. Cependant, il peut être utile d'utiliser une combinaison de températures sur plusieurs jours afin de tenir compte de l'inertie du bassin [9].

$S$ est généralement exprimé sous la forme d'un quotient permettant de suivre la quantité de stock disponible. Il peut tenir compte de la répartition des précipitations solides sur le bassin et du pourcentage de surface enneigée ([7], [8], [9] et [11]). Pour un petit bassin où toute la surface reste enneigée, la fusion ne peut que difficilement être reliée à la quantité de stock disponible et c'est plus l'altération du stock et l'effet des précipitations liquides en fin de fusion qui interviennent comme indice de fonte.

La comparaison des températures extérieures avec l'évolution du débit $Q^{n}$ liée aux apports de fusion du manteau (fig. 5) montre clairement des phénomènes de non linéarité et de non stationnarité du processus. En effet des températures extérieures positives de même intensité n'ont pas les mêmes conséquences sur les débits obtenus à la source de Chavanette. De plus, le décalage en temps entre les températures positives et les débits consécutifs est nettement plus important pour les deux premiers pics de crues de fin de tarissement (fin mars) que pour les débits liés à la fusion généralisée du manteau (à partir du 22 avril).
La relation utilisée entre les températures extérieures dépassant le seuil $T_{0}$ et les débits consécutifs doit donc tenir compte du décalage temporel entre ces derniers.

Le modèle proposé est donc de la forme:

$$
\begin{aligned}
Q_{d}^{n^{*}}=a\left(T_{d}-T_{0}\right)+b\left(T_{d-1}\right. & \left.-T_{0}\right)+ \\
& +c\left(T_{d-2}-T_{0}\right)+d\left(T_{d-3}-T_{0}\right)
\end{aligned}
$$

$Q_{d}^{n^{*}}$ : Débit lié à la fusion du manteau

$T_{d}$ : Température moyenne du jour $d$

$T_{0}$ : Seuil de température

Afin de tenir compte d'un phénomène d'inertie entre la température extérieure et le réchauffement du manteau, un pré-traitement des températures extérieures est établi de la façon suivante :

si $\quad \frac{T_{d}+T_{d-1}+T_{d-2}}{3}<-1,5^{\circ} \mathrm{C}$ alors $T_{d}=-1,5^{\circ} \mathrm{C}$

Ce pré-traitement a pour conséquence, en particulier, d'égaliser à $-1,5^{\circ} \mathrm{C}$ une température moyenne extérieure positive si les températures des deux jours précédents sont fortement négatives.

La valeur de $-1,5^{\circ} \mathrm{C}$ correspond par ailleurs au seuil de température moyenne du manteau au-dessus duquel il y a présence d'eau liquide (fig. 2).

Ce pré-traitement des données a amélioré l'optimisation des paramètres $a, b, c$ et $d$ obtenus par minimisation de la somme des carrés des résidus $S$ sur la période du 17 mars au 16 mai 1994 , c'est-à-dire :

$$
S=\sum_{i=1}^{n}\left(Q_{d}^{n^{*}}-Q_{d}^{n}\right)^{2}
$$

$Q_{d}^{n^{*}}$ : Débit simulé du jour $d$ lié à la fusion du manteau $Q_{d}^{n}$ : Débit théorique déterminé par le modèle de récession $n$ : Nombre de jours de la période de calage

L'optimisation, avec une contrainte de positivité des paramètres $a, b, c$ et $d$ afin qu'une différence positive entre la température moyenne journalière et le seuil $T_{0}$ ici égal à $-1{ }^{\circ} \mathrm{C}$ ne soit pas la conséquence d'un débit négatif, conduit à (fig. 6) :

$$
Q_{d}^{n^{*}}=0,28\left(T_{d}-1\right)+\left(T_{d-1}-1\right)+0,86\left(T_{d-2}-1\right)
$$

avec $S=83,7$

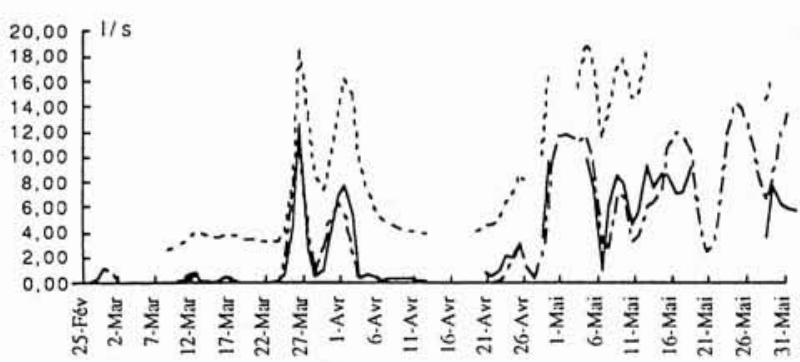

6. Comparaison du débit de fusion simulé (Tirets-points) avec le débit calculé à partir du modèle de récession (Trait plein) et débit total (Tirets) pour 1994. 
Cependant, une optimisation, établie indépendamment sur les deux premiers pics de débits de fin de tarissement (fin mars (a)) et sur les crues liées à la fusion généralisée (à partir de fin avril (b)), donne respectivement les relations suivantes (fig. 7):

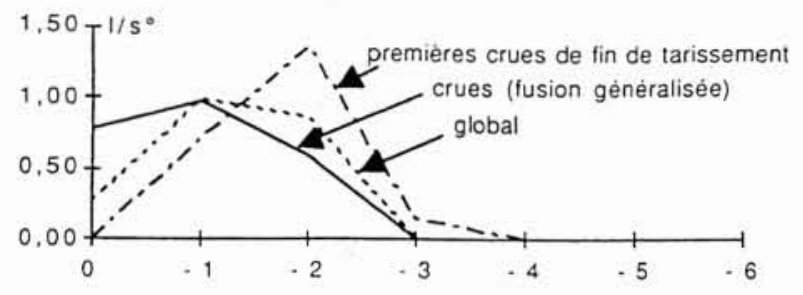

7. Variation des paramètres des relations température extérieure-débit de fusion optimisés sur les deux crues de fin d'étiage (Tirets-points), sur les crues de fusion généralisée (Trait plein) et sur toute la période (Tirets).

(a)

$Q_{d}^{n^{*}}=0,72\left(T_{d-1}-1\right)+1,35\left(T_{d-2}-1\right)+0,15\left(T_{d-3}-1\right)$ avec $S=2,7$

(b)

$Q_{d}^{n^{*}}=0,78\left(T_{d}-1\right)+0,96\left(T_{d-1}-1\right)+0,58\left(T_{d-2}-1\right)$

avec $S=19,5$

Ces résultats montrent que le processus qui relie les températures extérieures et les débits consécutifs est différent entre les deux périodes considérées avec un retard plus important pour les deux premières crues de fin de tarissement. Ce décalage peut être lié aux propriétés du manteau mais aussi à l'infiltration localisée d'eau de fonte lors des premiers réchauffements, ce qui confirmerait les observations précédentes faites sur le comportement de l'aquifère et de son alimentation.

\section{VIII $\square$ CONCLUSION}

L'étude d'un aquifère fissuré et calcaire de haute montagne a permis de caractériser un milieu présentant une faible réserve avec des vitesses de transfert rapides. Pour un système constitué d'un couvert neigeux et d'un écoulement souterrain, les réponses à la fusion nivale ont permis de montrer un comportement différent entre les premières crues de fin de tarissement et celles liées à la fusion généralisée. Cette différence s'observe au niveau de l'évolution des paramètres physico-chimiques de l'eau de source ainsi que sur la comparaison des décompositions d'hydrogrammes déterminés par différentes méthodes. Enfin pour ce type de système, un modèle de type degré-jour est appliqué sur un processus non stationnaire. La relation entre les températures extérieures et le débit à la source de Chavanette est améliorée en tenant compte d'un effet d'inertie entre le réchauffement de l'air et celui du manteau. Cependant les résultats sont encore différents lorsque l'on s'intéresse indépendamment aux premières crues de fin de tarissement et celles liées à la fusion généralisée.

Les auteurs remercient Messieurs Page, Subtil et Fachinger ainsi que Messieurs les Directeurs et le personnel de la Société Lyonnaise des Eaux-Dumez et du Service des Pistes de la SERMA pour leur apport scientifique, technique ou financier.

\section{Bibliographie}

[1] Gupta R.P., Duggal A.J., Rao S.N., Sankar G., Singhal B.B.S. (1982). - Snow-cover area vs. Snowmelt runoff relation and its dependence on geomorphology. A study from the beas catchment (Himalaya, India). J. Hydrol., 58, 325339.

[2] Bottomley D.J., Craig D., Johnston L.M. (1986). Oxygen-18 studies of snowmelt runoff in a small precambrien shield watershed: implications for streamwater acidification in acid-sensitive terrain. J. Hydrol., 88, 213-234.

[3] Maulé C.P., Stein J. (1990). - Hydrologic flow path definition and partitioning of spring meltwater. Water Resour. Res., 26(12), 2959-2970.

[4] Obradovic M.M., SkLash M.G. (1986). - An isotopic and geochimical study of snowmelt runoff in a small arctic watershed. Hydrological processes, 1, 15-30.

[5] KoBAYASHi D. (1985). - Separation of the snowmelt hydrograph by stream temperatures. J. Hydrol., 76, 155-162.

[6] KOBAYASHI D. 1986. - Separation of the snowmelt hydrograph by stream conductance. J. Hydrol., 84, 157-165.

[7] HiNO M., HASEBE M., NODA K. (1987). - Estimation of volume of snowmelt from temperature of snow line and residual snow amount. J. Hydrol., 93, 25-39.

[8] Martinec J., RANGo A. (1986). - Parameter values for snowmelt runoff modelling. J. Hydrol., 84, 197-219.

[9] RODRIGUEZ J.Y. (1993). - An operational forecasting snowmelt model with objective calibration. Nordic Hydrology, 25, 79-100.

[10] BONACCI O. (1993). - Karst springs hydrographs as indicators of karst aquifers. Hydrol. Sci. J., 38, 1, 51-63.

[11] Roche M., SLIVITZKY M. (1969). - Modèle mathématique pour une crue de fonte de neige, in Floods and their computation, IAHS Publ. 84-85, 688-699.

[12] MARTINEC J. (1960). - The degree-day factor for snowmelt forecasting, in Proceed. of Symposium General Assembly of IAHS. Helsinky. Finland, IAHS pub. 51, 127-134. 